\title{
Review on Dark Photon
}

\author{
Francesca Curciarello $1,2,3, \mathrm{a}$ \\ ${ }^{1}$ Novosibirsk State University, 630090 Novosibirsk, Russia \\ ${ }^{2}$ Dipartimento di Fisica e di Scienze della Terra, Università di Messina, Italy \\ ${ }^{3}$ INFN Sezione Catania, Italy
}

\begin{abstract}
. $\mathrm{e}^{+} \mathrm{e}^{-}$collider experiments at the intensity frontier are naturally suited to probe the existence of a force beyond the Standard Model between WIMPs, the most viable dark matter candidates. The mediator of this new force, known as dark photon, should be a new vector gauge boson very weakly coupled to the Standard Model photon. No significant signal has been observed so far. I will report on current limits set on the coupling factor $\varepsilon^{2}$ between the photon and the dark photon by $\mathrm{e}^{+} \mathrm{e}^{-}$collider experiments.
\end{abstract}

\section{Introduction}

The existence of a low energy dark sector, remained yet undiscovered because of the very small coupling, is predicted by many extensions of the Standard Model (SM) [1-5]. The popular paradigm is to consider a $100 \mathrm{GeV}$ - $10 \mathrm{TeV}$ Weak Interacting Massive Particle (WIMP) as the most viable dark matter candidate and assume that WIMPs are charged under a new weak-scale interaction. In principle, the postulation of a fifth force, under which SM particles are uncharged, is not related to the dark matter puzzle and dates back to the early 1980's [1]. Very recently, its search became a goal for the most important world facilities since it has been advocated as possible explanation of many puzzling astrophysical and terrestrial anomalies [6-12].

The mediator of this new force should be a neutral light vector gauge boson which can kinetically mix with the ordinary photon and thus should be produced in any process in which a virtual or real photon is involved. Coupling to SM photon would occur through loops of heavy dark particles, charged under both electroweak and dark interactions, giving rise to a very weak mixing strength defined as the ratio of dark and fine structure coupling constants $\left(\varepsilon^{2}=\alpha^{\prime} / \alpha<10^{-2}\right)$. Moreover, the dark photon (referred to also as $\mathrm{U}, A^{\prime}$ or $\gamma^{\prime}$ ) should get mass by means of a Higgs-like mechanism, suggesting the existence of the equivalent of the SM Higgs boson in the dark sector, the dark Higgs $h^{\prime}$. Dark photon search is also strongly motivated by particle physics since it should give a positive one loop contribution to the calculated value of the muon magnetic moment anomaly, $a_{\mu}$, solving the discrepancy for masses of $10-100 \mathrm{MeV}$ and coupling of $\varepsilon \sim 10^{-3}$ [13].

Thanks to the available huge data statistics and the good knowledge of backgrounds, high intensity flavour factories are well suited to probe dark forces [14] with

\footnotetext{
a e-mail: fcurciarello@unime.it
}

a wide and relatively inexpensive physic program. At $\mathrm{e}^{+} \mathrm{e}^{-}$colliders, dark forces searches are rich with possibilities and can be investigated by exploiting many different processes as radiative meson decays, continuum processes or dark Higgsstrahlung. In the minimal hypothesis of prompt and visible dark photon decays, the KLOE-2 experiment set many constraints by searching for the dark photon in the Dalitz decay $\Phi \rightarrow \eta \mathrm{U}, \mathrm{U} \rightarrow \mathrm{e}^{+} \mathrm{e}^{-}$and in the $\mathrm{e}^{+} \mathrm{e}^{-} \rightarrow \mathrm{U} \gamma$ continuum process, by considering both $\mathrm{U} \rightarrow$ $\mathrm{e}^{+} \mathrm{e}^{-}, \mu^{+} \mu^{-}$decay channels. Moreover, KLOE-2 investigated also the dark Higgsstrahlung process by assuming the invisible scenario, where $m_{\mathrm{U}}>m_{h^{\prime}}$ and the dark Higgs escapes detection showing up a missing energy. BaBar and Belle set complementary constraints on dark Higgsstrahlung by considering the visible scenario, $m_{\mathrm{U}}<m_{h^{\prime}}$, consisting in a multi-lepton or pion final state events. Furthermore, BaBar extracted a stringent constraint by combining limits obtained by using the Initial State Radiation (ISR) processes $\mathrm{e}^{+} \mathrm{e}^{-} \rightarrow \mathrm{U} \gamma, \mathrm{U} \rightarrow l^{+} l^{-}(l=\mathrm{e}, \mu)$ which ruled out most of the remaining $(g-2)_{\mu}$ favoured region assuming only the visible decay hypothesis.

I will review the status and future prospects of the limits on the mixing strength between the photon and the dark photon set at $\mathrm{e}^{+} \mathrm{e}^{-}$collider experiments.

\section{Dark forces at KLOE}

\section{1 $\Phi$ Dalitz decay}

The dark photon is expected to be produced in vector $(\mathrm{V})$ to pseudoscalar $(\mathrm{P})$ meson decays but with a rate $\varepsilon^{2}$ times suppressed with respect to the ordinary $\mathrm{V} \rightarrow \mathrm{P}$ transitions [15] (see Fig. 1). The branching ratio of the $\mathrm{U}$ boson into $\mathrm{e}^{+} \mathrm{e}^{-}$is non-negligible, thus, $\mathrm{V} \rightarrow \mathrm{P} \mathrm{U}$ events are expected to produce a peak in the invariant mass distribution of the electron-positron pair over the continuum Dalitz background. 


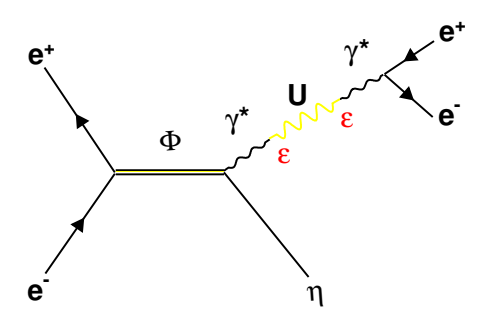

Figure 1. U-boson production through Dalitz $\phi$-meson decay.

KLOE-2 investigated this hypothesis setting two constraints on the U-boson coupling $\varepsilon^{2}$, by exploiting the $\phi \rightarrow \eta \mathrm{e}^{+} \mathrm{e}^{-}$decay, where the $\eta$ meson is tagged by its $\pi^{+} \pi^{-} \pi^{0}$ [16] and $3 \pi^{0}$ decays [17]. The first analysis used a data sample of $1.5 \mathrm{fb}^{-1}$ integrated luminosity with a $2 \%$ of background contamination. The limit on the number of U-boson events has been set by using the Confidence Level Signal $\left(\mathrm{CL}_{\mathrm{S}}\right)$ technique [18-20]. This first upper limit (UL) has been then updated, improving sample statistics and background rejection, and combined with a new limit derived by tagging the $\eta$ meson by its neutral decay into $3 \pi^{0}$ [17]. For this new analysis, 30577 events are selected from a data sample of $1.7 \mathrm{fb}^{-1}$ with $3 \%$ of residual background. For each channel, the irreducible background is extracted directly from data after applying a bin-by-bin subtraction of the non-irreducible backgrounds and correcting for the analysis efficiency. The final $M_{\mathrm{ee}}$ distribution is then fitted to estimate the expected background, excluding for each U-boson mass hypothesis, the possible signal region used for the upper limit evaluation (5 MeV centred around $m_{\mathrm{U}}$ ).

A combined UL on the parameter $\varepsilon^{2}$ at $90 \% \mathrm{CL}$ has been derived by using the Vector Meson Dominance expectation for the transition form factor slope $\left(b_{\phi \eta} \sim 1 \mathrm{GeV}^{2}\right)$ resulting in $\varepsilon^{2}<1.7 \times 10^{-5}$ for $30<M_{\mathrm{U}}<$ $400 \mathrm{MeV}$, and for the sub-region $50<M_{\mathrm{U}}<210 \mathrm{MeV}$, in $\varepsilon^{2}<8.0 \times 10^{-6}$. The above final combined limit is shown in Fig. 3 and Fig. 6. This limit, published on 2013 [17] was able to rule out a wide range of U-boson parameters as a possible explanation of the $a_{\mu}$ discrepancy in the minimal U-decay hypothesis.

\section{$2.2 \mathrm{U} \gamma$ events}

Radiative U-boson production in $\mathrm{e}^{+} \mathrm{e}^{-} \rightarrow \mathrm{U} \gamma, \mathrm{U} \rightarrow$ $l^{+} l^{-}, l=\mathrm{e}, \mu$ events is considered very promising since it is a clean and simple process and it is independent of the details of the Higgs sector of the dark group (see Fig. 2). The $\mathrm{U}$ boson should appear as a resonant peak in the invariant mass distribution of the lepton pair induced by the photon's radiative return mechanism [21]. KLOE investigated both the $\mathrm{U} \rightarrow \mu^{+} \mu^{-}$and $\mathrm{U} \rightarrow \mathrm{e}^{+} \mathrm{e}^{-}$hypotheses. The search for $\mathrm{U} \rightarrow \mu^{+} \mu^{-}$employed a data sample collected in 2002 at the DAФNE $\mathrm{e}^{+} \mathrm{e}^{-}$collider with an integrated luminosity of $239.3 \mathrm{pb}^{-1}$. Event selection required two tracks of opposite charge with $50^{\circ}<\theta<130^{\circ}$ and an undetected photon whose momentum points at small

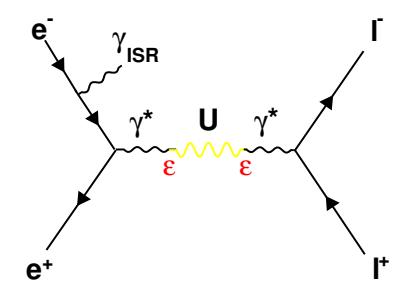

Figure 2. Resonant U-boson production in $\mathrm{U} \gamma$ events, $l=\mathrm{e}, \mu$.

polar angle $\left(\theta<15^{\circ},>165^{\circ}\right)[22,23]$. This event selection allowed to reduce the background coming from Final State Radiation and $\phi$-resonant processes. Moreover, since the final state $\gamma$ is emitted with high probability by the initial-state electron or positron, this increases sensitivity on $\mathrm{U} \rightarrow \mu^{+} \mu^{-}$decay. At the end of analysis selection, the differential cross section $\mathrm{d} \sigma_{\mu \mu \gamma} / \mathrm{dM}_{\mu \mu}$ has been derived achieving excellent agreement between the measurement and the simulation based on PHOKHARA [24]. No structures are visible in the $M_{\mu \mu}$ spectrum and a limit is extracted on the number of U-boson candidates through the $\mathrm{CL}_{\mathrm{S}}$ technique [18-20]. Since the agreement between data and simulation was below $1 \%$, the expected background for the limit extraction has been determined by using the MC PHOKHARA generation. The limit on the number of U-boson events has been converted in terms of the kinetic mixing parameter $\varepsilon^{2}$ by using the formula reported in Ref. [23]. The limit on $\varepsilon^{2}$ results in $1.6 \times 10^{-5}$ and 8.6 $\times 10^{-7}$ in the 520-980 MeV energy range.

The KLOE search for the $\mathrm{U} \rightarrow \mathrm{e}^{+} \mathrm{e}^{-}$employed a data sample of an integrated luminosity of $1.54 \mathrm{fb}^{-1}$. In this analysis the hard ISR photon has been explicitly detected in the calorimeter barrel by requiring a polar angle $50^{\circ}<\theta<30^{\circ}$ for the charged leptons and photon. This large-angle event selection allowed to have sufficient statistics to reach the dielectron mass threshold. At the end of the analysis chain the background contamination is less than $1.5 \%$. Also in this case no resonant U-boson peak was observed and again the $\mathrm{CL}_{\mathrm{S}}[18-20]$ technique is applied to estimate U-boson signal events excluded at $90 \%$ CL. A limit on the kinetic mixing parameter as a function of $m_{\mathrm{U}}$ was set by using the same equation reported in Refs. [23] and [25]. The resulting exclusion plot is shown in Fig. 3 with all other existing limits in the region 0-1000 MeV [16, 17, 23, 25-31]. The new KLOE limit excludes some of the remaining $g-2$ favoured region (see yellow line in Fig. 3) and has been recently published [25].

\subsection{Higgsstrahlung process}

If the $\mathrm{U}$ boson exists it natural to conceive the breaking of the additional $U_{D}(1)$ symmetry by a Higgs-like mechanism. In this case an additional scalar particle, the dark Higgs h' [32] should exist as well. This suggests to probe the processes shown in Figs. 4 and 7. The Higgsstrahulung process is of particular interest since it is also sensitive to the dark coupling constant $\alpha_{\mathrm{D}}=G_{\mathrm{D}} / 4 \pi\left(G_{\mathrm{D}}\right.$ dark gauge coupling) and, in contrast to the above shown processes, 


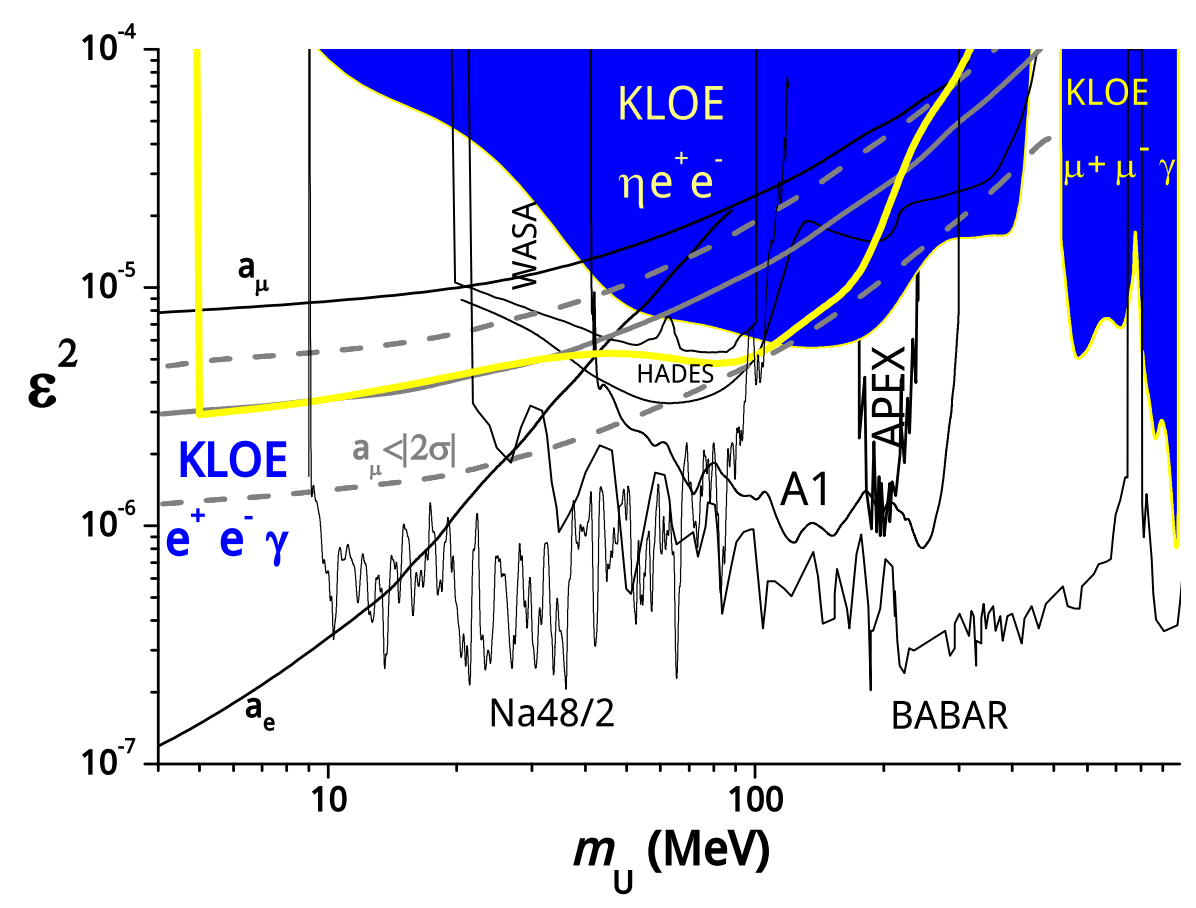

Figure 3. $90 \% \mathrm{CL}$ exclusion plot for $\varepsilon^{2}$ as a function of the U-boson mass. The KLOE limits from $\Phi$ Dalitz dacay [16, 17], $\mathrm{e}^{+} \mathrm{e}^{-} \rightarrow \mathrm{U} \gamma \rightarrow \mu^{+} \mu^{-}\left(\mathrm{e}^{+} \mathrm{e}^{-}\right) \gamma$ [23, 25] and from BaBar [26], the limits from the A1 [27] and Apex [28] fixed-target experiments, the WASA [29], HADES [30] and NA48/2 [31] limits are also shown. The solid lines are the limits from the muon and electron anomaly [13], respectively. The gray line shows the U-boson parameters that could explain the observed $a_{\mu}$ discrepancy with a $2 \sigma$ error band (gray dashed lines) [13].

it is suppressed by only a single factor of $\varepsilon^{2}$. As for the SM Higgs, the mass of the dark Higgs is not predicted by theory so different scenarios are possible according to $U$ boson and dark Higgs mass hierarchy.

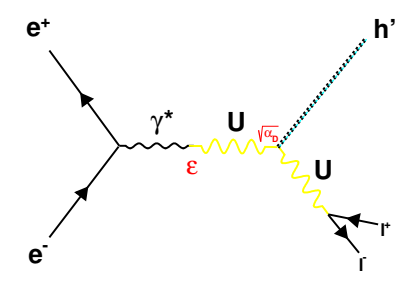

Figure 4. Dark Higgsstrahlung process in the invisible scenario with a long lived dark higgs $\mathrm{h}^{\prime}$ which escapes detection producing a missing energy signal $(l=\mathrm{e}, \mu, \pi)$.

If the $\mathrm{h}^{\prime}$ is lighter than the $\mathrm{U}$ boson, it turns out to be very long-lived and escapes detection (see Fig. 4). In this case, the expected signal will be a lepton or pion pair from the U-boson decay plus missing energy.

KLOE studied the Higgsstrahlung process in the energy range $2 m_{\mu}<M_{\mathrm{U}}<1000 \mathrm{MeV}$, by considering the process $e^{+} e^{-} \rightarrow \mathrm{h}^{\prime} \mathrm{U}, \mathrm{U} \rightarrow \mu^{+} \mu^{-}, \mathrm{h}^{\prime}$ invisible [33]. The analysis has been performed by using a data sample of $1.65 \mathrm{fb}^{-1}$ collected at center- of-mass energy $E_{\mathrm{cm}}$ corre-

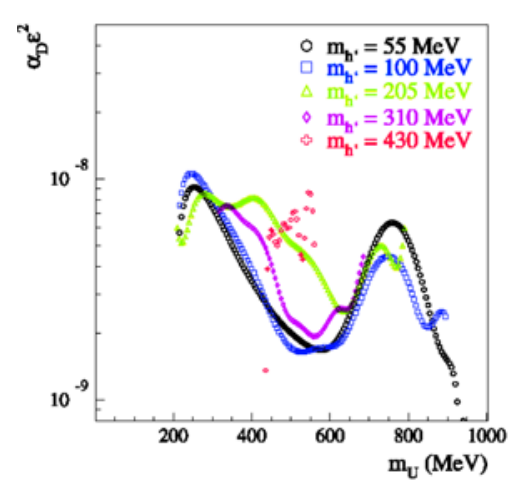

Figure 5. 90\% CL combined upper limits in $\alpha_{\mathrm{D}} \times \varepsilon^{2}$ as a function of $m_{\mathrm{U}}$ for different values of $m_{\mathrm{h}^{\prime}}$.

sponding to the $\phi$ peak $(\sim 1019 \mathrm{MeV})$ and a data sample of $0.2 \mathrm{fb}^{-1}$ at $E_{\mathrm{cm}}=1000 \mathrm{MeV}$ (off-peak sample).

The expected signal would appear in the $M_{\mu \mu}-M_{\text {miss }}$ bi-dimensional spectra. No signal signature has been observed and a Bayesian limit on the number of signal events at $90 \%$ CL has been evaluated, bin-by-bin, for the on-peak and off-peak sample separately. Results have been translated in terms of $\alpha_{\mathrm{D}} \times \varepsilon^{2}$ by using the integrated luminosity information, the signal efficiency, the dark Higgsstrahlung cross section and the branching fraction of the $U \rightarrow \mu^{+} \mu^{-}$ 


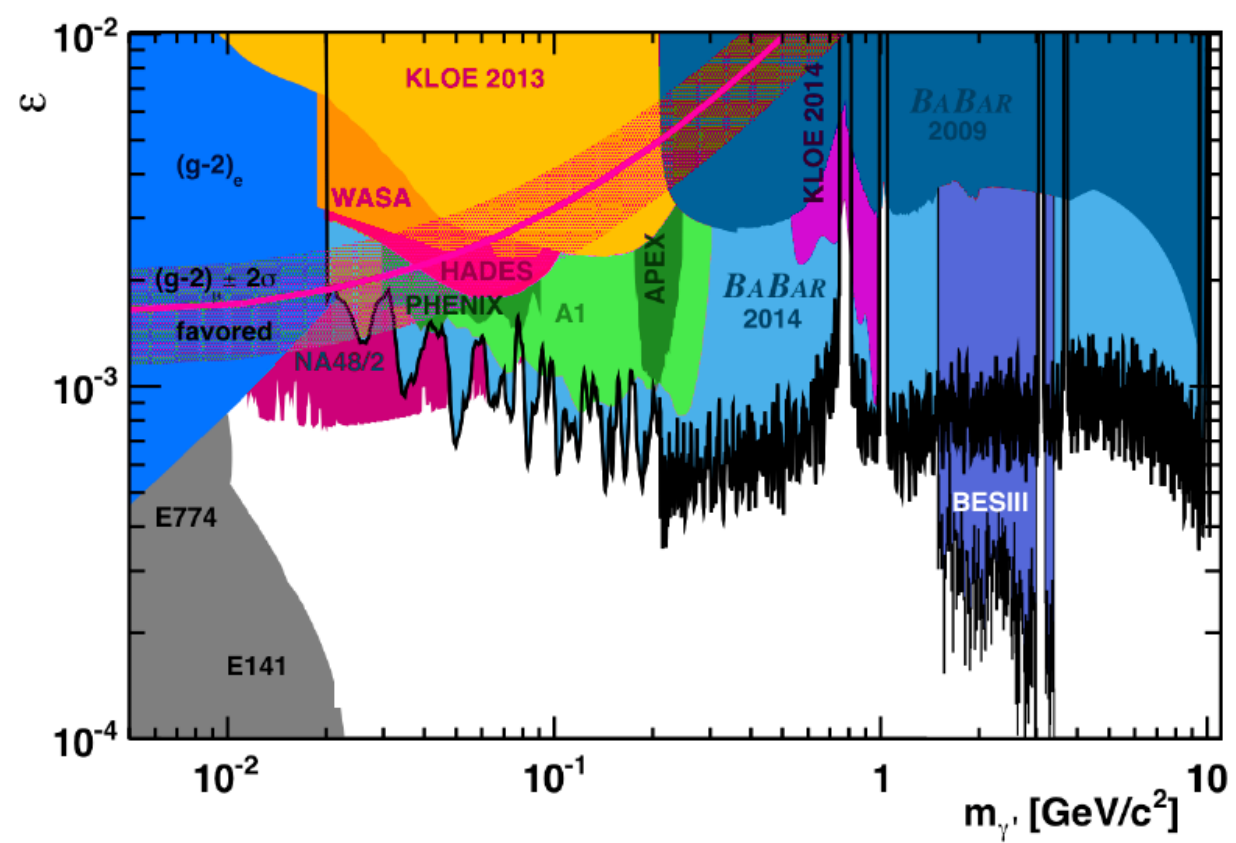

Figure 6. 90\% CL currents limits on epsilon (for details see Fig. 3) up to $10 \mathrm{GeV}$. The preliminary limit from BES-III presented at the CHARM 2015 Conference is also shown [40].

decay [32]. The combined upper limits projected in the $M_{\mu \mu}$ invariant mass, for different dark Higgs mass hypotheses and slightly smoothed, are shown in Fig. 5. Values of the order of $10^{-9} \div 10^{-8}$ in $\alpha_{\mathrm{D}} \times \varepsilon^{2}$ are excluded at $90 \% \mathrm{CL}$ for a large range of the dark photon and dark Higgs masses. These limits translate in $\varepsilon \sim 10^{-3}-10^{-4}$ for $\alpha_{\mathrm{D}}=\alpha$ and are in agreement and complementary with BaBar results [34] as they refer to the same process in a different final state and phase space region.

\section{Dark forces at BaBar}

\section{1 $\mathrm{U} \gamma$ events}

BaBar also exploited ISR to search for dark photon. The analysis has been performed on a data sample of about $514 \mathrm{fb}^{-1}$ collected mainly at $\sqrt{s} \sim \Upsilon(4 s)$ but also at $\Upsilon(2 s)$ and $\Upsilon(3 s)$ resonances as well as in the regions in between these resonances, exploring the mass range $0.02<$ $m_{\mathrm{U}}<10.2 \mathrm{GeV}$. Event selection required two oppositely charged tracks plus a photon having a center-of-mass energy greater than $0.2 \mathrm{GeV}$. For the dielectron channel, the background processes $\left(\mathrm{e}^{+} \mathrm{e}^{-} \rightarrow \gamma \gamma(\gamma), \mathrm{e}^{+} \mathrm{e}^{-} \rightarrow \mathrm{e}^{+} \mathrm{e}^{-} \gamma\right)$ have been simulated with BHWIDE [35], while simulation of $\mathrm{e}^{+} \mathrm{e}^{-} \rightarrow \mu^{+} \mu^{-} \gamma$ events has been performed with KKMC [36]. A very good agreement is achieved between data and MC simulation for both channels. The disagreement below $1 \mathrm{GeV}$ between radiative Bhabha data and simulation is due to MC cut-off and does not affect limit extraction since for both channels the expected background has been derived directly by a fit to data. Uncertainty on expected background modelling is below the statistical uncertainties in the whole investigated range. $90 \%$ CL Bayesian upper limits on the $\mathrm{e}^{+} \mathrm{e}^{-} \rightarrow \mathrm{U} \gamma$ cross-section have been derived (typically $O(1-10) \mathrm{fb}$ ), assuming a flat prior for the cross-section. Resonance regions $( \pm 30 \mathrm{MeV}$ around $\rho / \omega, \pm 50 \mathrm{MeV}$ around $J / \Psi, \Psi(2 s)$ and $\Upsilon(1 s, 2 s)$ ) are excluded from the limit extraction. Results are finally translated into 90\% CL upper limits on the mixing strength between the photon and dark photon as a function of the dark photon mass. The final combined $\mathrm{BaBar}$ constraint is shown in Fig. 6, set bounds are at the level of $10^{-3}-10^{-4}$. This strong limit [26] improves results from many experiments and further constrains the $g-2$ favoured region.

\subsection{Higgsstrahlung process}

If the dark Higgs has a mass greater than two U-boson masses, then the dark Higgs will decay into a couple of $\mathrm{U}$ bosons. In this case, possible final state signatures consist of six particles (inclusive channels) or of four leptons plus a $\mathrm{U}$ boson which escapes detection and is detected via missing mass (exclusive channels), see Fig. 7.

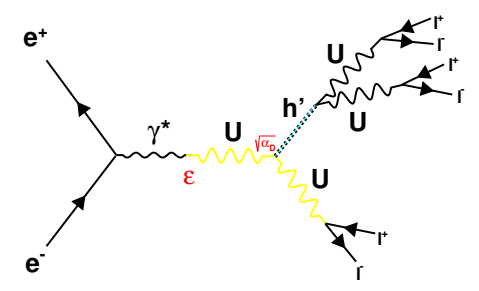

Figure 7. Dark Higgsstrahlung process in the visible scenario; since $m_{\mathrm{h}^{\prime}}>2 m_{\mathrm{U}}$ final states signature consists of six-particle events (exclusive channels) or of four-lepton events plus missing energy (inclusive channels), $l=e, \mu, \pi$. 
BaBar investigated the Higgsstrahlung process by assuming prompt decays for both dark photon and dark Higgs and by considering $m_{\mathrm{h}^{\prime}}>2 m_{\mathrm{U}}$. Full BaBar statistics, about $521 \mathrm{fb}^{-1}$, has been exploited to fully reconstruct the $\mathrm{e}^{+} \mathrm{e}^{-} \rightarrow \mathrm{h}^{\prime} \mathrm{U}, \mathrm{h}^{\prime} \rightarrow$ UU reaction in the $3\left(l^{+} l^{-}\right), 2\left(l^{+} l^{-}\right)+\pi^{+} \pi^{-}, 2\left(\pi^{+} \pi^{-}\right)+l^{+} l^{-}$exclusive final states or partially reconstruct it in the $2\left(\mu^{+} \mu^{-}\right)+$ $\mathrm{X}, \mu^{+} \mu^{-} \mathrm{e}^{+} \mathrm{e}^{-}+\mathrm{X}$ inclusive final states, where $\mathrm{X}$ denotes any final state other than a pair of pions or leptons while $l=\mathrm{e}, \mu$. The $2\left(\mathrm{e}^{+} \mathrm{e}^{-}\right)+\mathrm{X}$ final state has been excluded because of huge background. Six candidates passed the event selection: $4 \pi+2 l, 4 \mu+2 \pi$ and $4 \mu+\mathrm{X}$. No sixlepton final-state events survived selection criteria and no significant signal is observed in the investigated range $\left(0.25<m_{\mathrm{U}}<3 \mathrm{GeV}, 0.8<m_{\mathrm{h}^{\prime}}<10 \mathrm{GeV}\right) .90 \% \mathrm{CL}$ upper limits have been set on $\alpha_{\mathrm{D}} \times \varepsilon^{2}$ at the level of $10^{-8}$ $10^{-10}$ (see Fig. 8) which for $\alpha=\alpha_{\mathrm{D}}$ translate in a limit on $\varepsilon$ of $10^{-3}-10^{-4}$.

\section{Dark forces at Belle}

\subsection{Higgsstrahlung process}

Recently Belle also studied the Higgsstrahlung in the same hypotheses of BaBar (visible scenario and prompt decay regime, see Fig. 7). By exploiting the full data set $\left(977 \mathrm{fb}^{-1}\right)$, Belle investigated ten exclusive final states: $3\left(l^{+} l^{-}\right), 2\left(l^{+} l^{-}\right)+\pi^{+} \pi^{-}, 2\left(\pi^{+} \pi^{-}\right)+l^{+} l^{-}$, and $3\left(\pi^{+} \pi^{-}\right)$in the $0.1<m_{\mathrm{U}}<3.5 \mathrm{GeV}$ and $0.2<m_{\mathrm{h}^{\prime}}<10.5 \mathrm{GeV}$ ranges, and three inclusive final states: $2\left(\mathrm{e}^{+} \mathrm{e}^{-}\right)+\mathrm{X}, 2\left(\mu^{+} \mu^{-}\right)+$ $\mathrm{X}, \mathrm{e}^{+} \mathrm{e}^{-} \mu^{+} \mu^{-}+\mathrm{X}$ for $m_{\mathrm{U}}>1.1 \mathrm{GeV}$ and $2.5<m_{\mathrm{h}^{\prime}}<10.5$ $\mathrm{GeV}$. Individual and combined $90 \%$ CL Bayesian upper limits on the branching fraction times the Born cross section, $\mathcal{B} \times \sigma_{\text {Born }}$, and on the Born cross section, $\sigma_{\text {Born }}$, have been derived and translated in limits on $\alpha_{\mathrm{D}} \times \varepsilon^{2}$. These final limits, shown in Fig. 8, improve upon and explore wider mass ranges than the $\mathrm{BaBar}$ experiment. The limits from the final states $3\left(\pi^{+} \pi^{-}\right)$and $2\left(\mathrm{e}^{+} \mathrm{e}^{-}\right)+\mathrm{X}$ are the first placed by any experiment [37]. For $\alpha_{\mathrm{D}}=\alpha, m_{\mathrm{h}^{\prime}}<8 \mathrm{GeV}$, and $m_{\mathrm{U}}<1 \mathrm{GeV}$, the above limits translate in limits on the mixing parameter, $\varepsilon$ of about $8 \times 10^{-4}$.

\section{Future Prospects}

Electron-positron colliders played a leading role in dark force searches and they are expected to play a crucial role in the near future as well, performing new searches and improving above shown limits. The current available data set has not been totally exploited regarding dark forces and new huge amount of data will be soon available thanks to the next data-taking campaigns.

The KLOE-2 run at LNF has started, taking advantage of new sub-detectors and new DAФNE interaction scheme, with the goal of reaching, within a few years, about $5 \mathrm{fb}^{-1}$. Moreover, at LNF a planned dedicated fixedtarget experiment, PADME [38], will be able to perform a model-independent dark photon search and to probe both visible and invisible decay hypotheses.

The Belle and BaBar experiments in Japan and USA have integrated about $1 \mathrm{ab}^{-1}$ each at $\sqrt{s} \sim 10 \mathrm{GeV}$. The aim is to reach $\sim 50 \mathrm{ab}^{-1}$ with future generation SuperB factories. With the implementation of a mono-photon trigger Belle-II will be also able to search for an invisibly decaying dark photon [39]. At $\sqrt{s} \sim 3 \mathrm{GeV}$, the BESIII detector in Beijing, China, aims to collect an integrated luminosity of $\sim 20 \mathrm{fb}^{-1}$, dark force searches are also in the program. A new preliminary combined limit on $\mathrm{U} \rightarrow \mathrm{e}^{+} \mathrm{e}^{-}, \mu^{+} \mu^{-}$decays has already been extracted in the 1.5-3.5 GeV energy range [40], see Fig. 6.

Moreover, it has to be noticed that null results obtained so far on dark photon searches are based on modeldependent searches which assumed only dark photon decays into SM particles. Most of the current set limits are no more valid in a more complex theoretical scenario with a dark photon decaying into light dark matter states. Concerning this non-minimal hypothesis, the $(g-2)_{\mu}$ favoured region can not be considered completely constrained [39]. In order to rule out dark photon as explanation of the muon anomaly or rule out it completely, a complete series of experiments, exploiting all theoretical possibilities, is needed.

\section{Conclusions}

Electron-positron colliders are naturally suited to search for dark forces, however no significant signal has been observed so far. New generation machines with their high statistics datasets would play a leading role by performing model-independent searches which are needed to solve dark force puzzle. Fixed target experiments will be more powerful to probe lower masses and very small couplings for which dark photon decay is no more prompt and its signature can be found by performing displaced vertex searches.

A signal should be observed in the near future, otherwise a change of the WIMP paradigm will be needed.

\section{References}

[1] B. Holdom, Phys. Lett. B 166, 196 (1985)

[2] C. Boehm and P. Fayet, Nucl. Phys. B 683, 219 (2004)

[3] P. Fayet, Phys. Rev. D 75, 115017 (2007)

[4] Y. Mambrini, J. Cosmol. Astropart. Phys. 1009, 022 (2010)

[5] M. Pospelov, A. Ritz and M.B. Voloshin, Phys. Lett. B 662, 53 (2008)

[6] O. Adriani et al., Nature 458, 607 (2009)

[7] M. Aguilar et al., Phys. Rev. Lett. 110, 141102 (2013)

[8] P. Jean et al., Astronomy Astrophysics 407, L55 (2003)

[9] J. Chang et al., Nature 456, 362 (2008)

[10] F. Aharonian et al., Phys. Rev. Lett. 101, 261104 (2008)

[11] A. A. Abdo et al., Phys. Rev. Lett. 102, 181101 (2009)

[12] R. Barnabei et al., Eur. Phys. J. C 56, 333 (2008) 

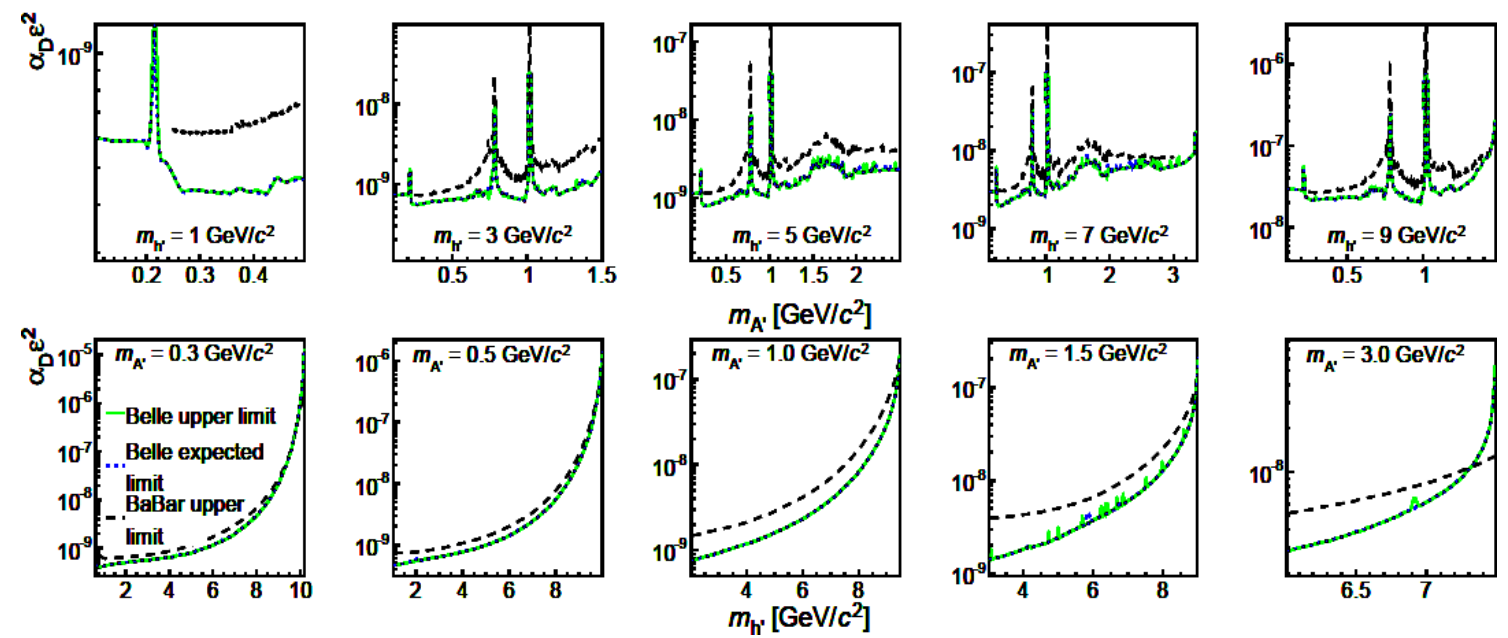

Figure 8. 90\% CL upper limit on the product $\alpha_{\mathrm{D}} \times \varepsilon^{2}$ versus dark photon mass (top row) and dark Higgs boson mass (bottom row) for Belle (solid green curve) and BaBar (dashed black curve [34]). The blue dotted curve shows the expected Belle limit.

[13] M. Pospelov, Phys. Rev. D 80, 095002 (2009)

[14] R. Essig, P. Schuster and N. Toro, Phys. Rev. textbf80, 015003 (2009)

[15] M. Reece, L.T. Wang, JHEP 07, 051 (2009)

[16] F. Archilli et al., Phys. Lett. B 706, 251 (2012)

[17] D. Babusci et al., Phys. Lett. B 720, 111 (2013)

[18] G. J. Feldman and R. D. Cousins, Phys. Rev. D 57, 3873 (1998)

[19] T. Junk, Nucl. Instr. Meth. A 434, 435 (1999)

[20] A. L. Read, J. Phys. G: Nucl. Part. Phys. 28, 2693 (2002)

[21] L. Barzè et al., Eur. Phys. J. C 71, 1680 (2011)

[22] D. Babusci et al., Phys. Lett. B 720, (2013) 336

[23] D. Babusci et al., Phys. Lett. B 736, (2014) 459

[24] H. Czyż , A. Grzelinska, J.H. Kühn, G. Rodrigo, Eur. Phys. J. C 39, (2005) 411

[25] A. Anastasi et al., Phys. Lett. B, 750, 633 (2015)

[26] J. P. Lees et al., Phys. Rev. Lett. 113, 201801 (2014)

[27] H. Merkel et al., Phys. Rev. Lett. 112, 221802 (2014)
[28] S. Abrahamyan et al., Phys. Rev. Lett. 107, 191804 (2011)

[29] P. Adlarson et al., Phys. Lett. B 726, 187 (2013)

[30] G. Agakishiev et al., Phys. Lett. B 731, 265 (2014)

[31] J. R. Batley et al., Phys. Lett. B 746, 178 (2015)

[32] B. Batell et al., Phys. Rev. D 79, 11508 (2009)

[33] A. Anastatsi et al., Phys. Lett. B 747, 365 (2015)

[34] J. P. Lees et al., Phys. Rev. Lett. 108, 211801 (2012)

[35] S. Jadach, W. Placzek and B. F. L. Ward Phys. Lett. B 390, 298 (1997)

[36] S. Jadach, W. Placzek and Z. Was, Phys. Rev. D 63, 113009 (2001)

[37] I. Jaegle et al., Phys. ReV. Lett. 114, 211801 (2015)

[38] M. Raggi and V. Kozhuharov Advances in High Energy Physics 2014, ID 959802 (2014)

[39] R. Essig et al., arXiv:1309.5084[hep-ph] (2015)

[40] http://docbes3.ihep.ac.cn/ talks/ images/c/cc/CHARM2015-prasad.pdf 